\title{
Occurrence of symptoms after catheter ablation of atrial fibrillation
}

\author{
Bou Ezzeddine H, Vachulova A, Svetlosak M, Urban L, Hlivak P, Margitfalvi P, Bernat V, \\ Gladisova K, Sasov M, Hatala R
}

Division of Academic Cardiology and Angiology, Medical Faculty, Slovak Medical University and National Cardiovascular Institute, Bratislava, Slovakia. hilda.bouezzeddine@gmail.com

\begin{abstract}
OBJECTIVES: The aim of our study was to verify the efficiency of catheter ablation of atrial fibrillation (CA AF) according to the "atrial fibrillation (AF) burden"(time spent in AF) and symptoms related to AF.

METHODS: We retrospectively analysed a selected set of 133 patients with atrial fibrillation (81\% men, 19\% women) who underwent an invasive therapy in the form of CA AF and at the same time had an implanted longterm ECG loop recorder (Reveal XT) in a period of eight years. We investigated AF burden and objective symptoms of AF by data obtained from a long-term implantable ECG loop recorder. Subjective symptoms related to AF were identified during outpatient controls.

RESULTS: Firstly, our results demonstrate for the first time a clinically relevant increase in the occurrence of asymptomatic episodes of AF after CA AF. Secondly, when analysing AF symptoms and AF burden at the same time, CAAF in terms of reduction of symptoms and shortening the time in AF had a better effect in patients undergoing 1 procedure (CA AF) compared to patients undergoing repeated procedures (re CA AF).

CONCLUSION: The increase in the occurrence of asymptomatic episodes of AF is of considerable importance both for the clinical evaluation of ablation efficacy and for individualized clinical management of patients, especially with respect to antithrombotic therapy (Fig. 10, Ref. 19). Text in PDF www.elis.sk.

KEY WORDS:atrial fibrillation, symptoms of atrial fibrillation, atrial fibrillation burden, catheter ablation of atrial fibrillation, continuous ECG monitoring.
\end{abstract}

\section{Introduction}

Atrial fibrillation (AF) is a cardiac arrhythmia with a high prevalence in the population and its incidence increases with age $(1,2,3)$. Catheter ablation of atrial fibrillation (CA AF) is an effective non-pharmacological treatment, which is used primarily for the treatment of AF in symptomatic patients with paroxysmal AF, with minimal structural heart disease in cases of resistance to antiarrhythmic therapy and patient preference. In line with the latest recommendations CA AF is assessed in the class IA indication when performed by an experienced physician (4). Continuous ECG monitoring is a valuable tool for assessing the exact duration of atrial fibrillation and at the same time for the objective evaluation of symptoms related to AF. European Heart Rhythm Association (EHRA) uses a scoring system (named also EHRA) that assesses symptoms attributable to AF and response

Division of Academic Cardiology and Angiology, Medical Faculty, Slovak Medical University and National Cardiovascular Institute - NUSCH, Department of Arrhythmias and Pacing, Bratislava, Slovakia

Address for correspondence: H. Bou Ezzeddine, MD, Division of Academic Cardiology and Angiology, Medical Faculty, Slovak Medical University and National Cardiovascular Institute - NUSCH, Department of Arrhythmias and Pacing, Pod Krásnou hôrkou 1, SK-833 48 Bratislava 37, Slovakia

Phone: +421.2.59320111 to therapy in terms of restoration of sinus rhythm or ventricular rate control (5).

$\mathrm{AF}$ is dangerous, because it can run as "silent $\mathrm{AF}^{\text {", }}$, that is $\mathrm{AF}$, which is asymptomatic. An asymptomatic $\mathrm{AF}$ is a frequent condition that can be found in up to $50 \%$ of evaluated patients and also in patients with a history of symptomatic atrial fibrillation $(6,7,8)$ This applies especially to patients suffering from paroxysmal AF in whom sustained asymptomatic AF occurs far more frequently than symptomatic AF (9) Patients who presented themselves as highly symptomatic of AF before catheter ablation of atrial fibrillation had a greater amount of asymptomatic episodes of AF after the procedure and this fact was rated as an improvement in "quality of life" by the patients $(10,11,12,13)$ On the other hand, repetition of AF with the "AF burden" (time spent in AF) lasting more than 12 hours over 3 months is not associated with improvements in quality of life. Moreover, symptomatic early return of AF predicts the return of AF independent of the type of treatment used (14)

The aim of our study was to assess the effectiveness of CA $\mathrm{AF}$ in terms of reduction of AF duration and symptoms related to AF by a retrospective analysis in a selected Slovak population of patients who underwent at least one CA AF and were implanted with a long-term ECG Holter monitor at the same time.

We enrolled 133 selected patients $(55 \pm 9$ years at the time of the first catheter ablation of atrial fibrillation - CA AF 1) $(81 \%$ males, $19 \%$ females) hospitalized at the Department of arrhythmias 
461-464

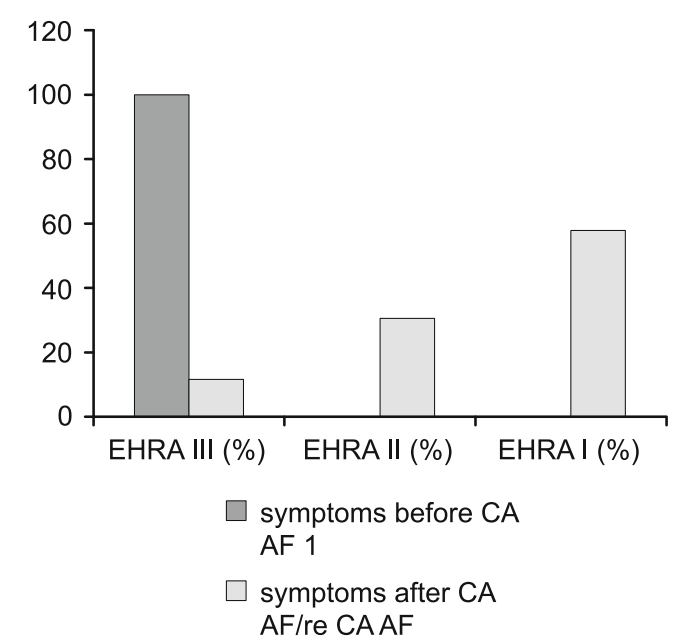

Fig. 1. Symptoms of AF patients undergoing CAAF before and after the procedure (s) (EHRA - evaluation of symptoms related to AF).

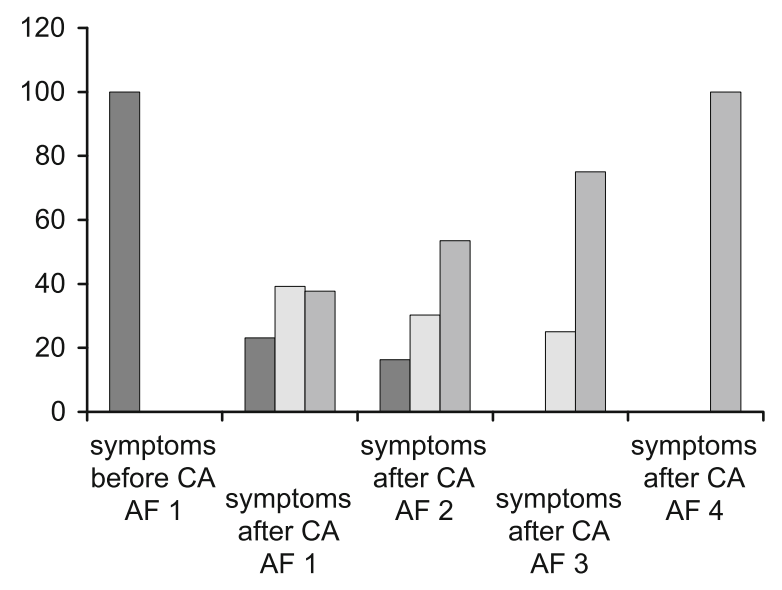

$\square$ EHRA III (\%) $\square$ EHRA II (\%) $\square$ EHRA I (\%)

Fig. 2. Symptoms of AF patients undergoing CAAF before and after the procedure (s) - detail (CA AF 1 - the first procedure of catheter ablation of atrial fibrillation, CAAF 2 - the second procedure of catheter ablation of atrial fibrillation, CA AF 3 - the third procedure of catheter ablation of atrial fibrillation, CA AF 4 - the fourth procedure of catheter ablation of atrial fibrillation) (EHRA - evaluation of symptoms related to $\mathrm{AF}$ ).

and pacing of the National Institute of Cardiovascular Diseases in Bratislava from October 2005 to January 2014. CA AF was performed as described elsewhere in detail and we used circumferential ablation (which has proven to be more effective than segmental ostial catheter ablation) of pulmonary veins with additional linear lesions when appropriate $(15,16,17)$ The patients' data were gained retrospectively from the hospital information system acquired during hospitalization, from subsequent outpatient monitoring and the specific interrogation programmer to long-term ECG loop recorder.

Descriptive statistics was used to analyse data. Continuous variables were described as mean value and standard deviation and discrete variables by chi-square test. Results are stated in graphs mainly as a percentage of a group of patients. Patients whose data were not available at the time of the study have been excluded.

Firstly, we studied the distribution of patients according to AF related symptoms before and after $\mathrm{CAAF}$ (or re $\mathrm{CAAF}$ - repeated CA AF, where appropriate). Patients were labelled as follows: highly symptomatic (EHRA III), mildly symptomatic (EHRA II) and asymptomatic (EHRA I). All patients presented themselves as highly symptomatic prior to CAAF. With an increasing number of procedures the number of highly symptomatic patients decreased in favour of mildly symptomatic patients and especially asymptomatic patients (11) documented the same results (Figs 1 and 2).

Secondly, all patients were divided according to the value of their last $\mathrm{AF}$ burden assessed after $\mathrm{CA} \mathrm{AF}$ into two groups: $\mathrm{AF}$ burden $>0.1 \%$ and AF burden $\leq 0.1 \%$. Afterwards patients in those groups were subdivided into groups of highly symptomatic patients (EHRA III), oligosymptomatic patients (EHRA II) and asymptomatic patients (EHRA I). Patients with AF burden $>0.1 \%$ and patients with AF burden $\leq 0.1 \%$ were represented about equally. Comparing a group of patients with $\mathrm{AF}$ burden $>0.1 \%$ and patients with AF burden $\leq 0.1 \%$ on the basis of symptoms associated with $\mathrm{AF}$, we found that in a group of patients with $\mathrm{AF}$ burden $\leq 0.1 \%$ no patient was highly symptomatic and most patients were represented in the EHRA class I (Figs 3, 4 and 5).

Thirdly, we divided the patients into two groups: patients undergoing one procedure (1 $\mathrm{CA} \mathrm{AF}$ ) and patients undergoing repeated procedures (re CA AF ( $\geq 2 \mathrm{CAAF})$ ) and those patients' groups were evaluated according to the symptoms and $\mathrm{AF}$ burden before and after the procedure (procedures).

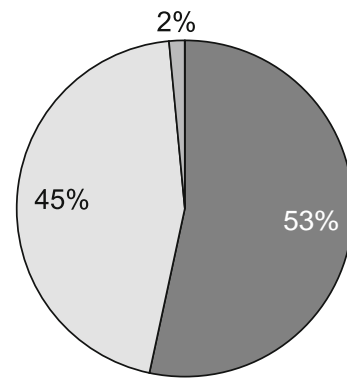

AF burden $>0.1 \%$

AF burden $\leq 0.1 \%$

AF burden NA

Fig. 3. Distribution of patients based on the value of the last AF burden (NA - not available).

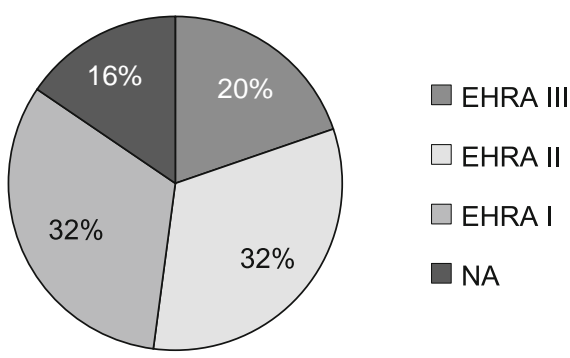

Fig. 4. Distribution of patients with AF burden $>0.1 \%$ according to their last symptomatology (EHRA - evaluation of symptoms related to AF, NA - not available). 


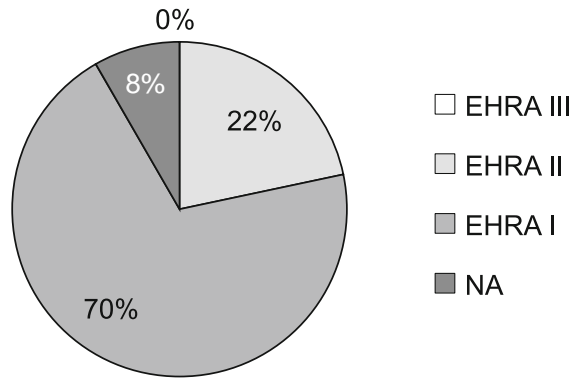

Fig. 5. Distribution of patients with $\mathrm{AF}$ burden $\leq 0.1 \%$ according to their last symptomatology (EHRA - evaluation of symptoms related to AF, NA - not available).

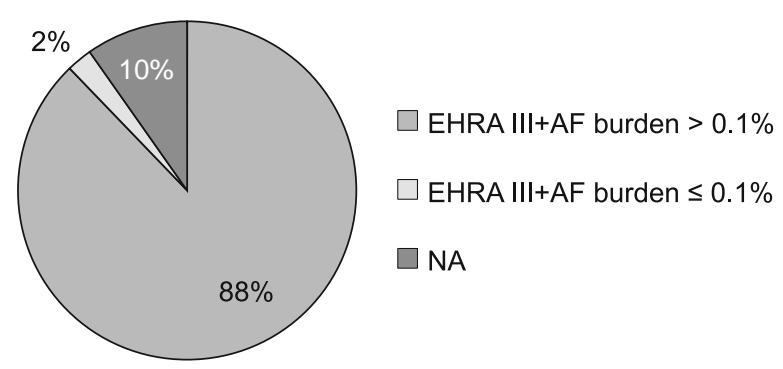

Fig. 6. Distribution of patients with one CA AF according to the AF symptoms and AF burden before the first CA AF (EHRA - evaluation of symptoms related to $\mathrm{AF}, \mathrm{NA}$ - not available).

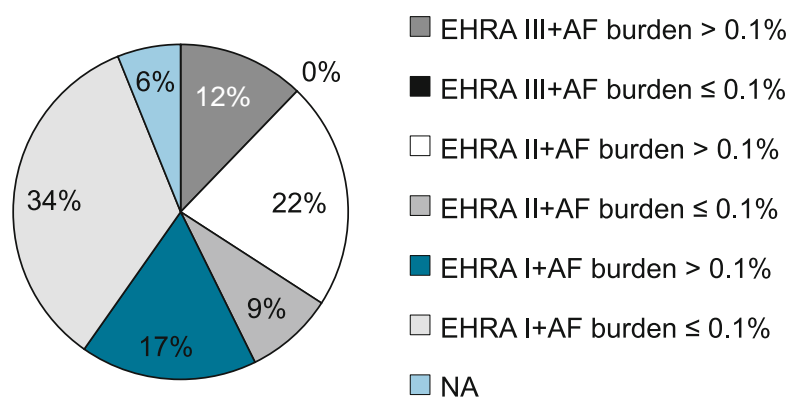

Fig. 7. Distribution of patients with one CA AF according to the AF symptoms and AF burden after the first CA AF (EHRA - evaluation of symptoms related to AF, NA - not available).

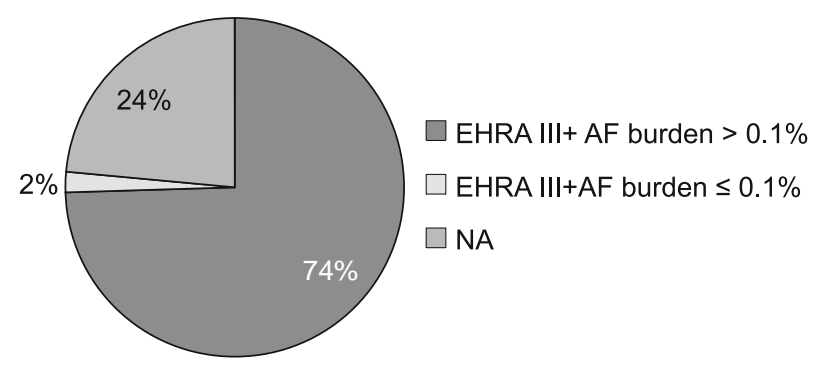

Fig. 8. Distribution of patients with $\geq 2 \mathrm{CA} A \mathrm{AF}$ according to the $\mathrm{AF}$ symptoms and AF burden before the first CA AF (EHRA - evaluation of symptoms related to $\mathrm{AF}, \mathrm{NA}$ - not available).

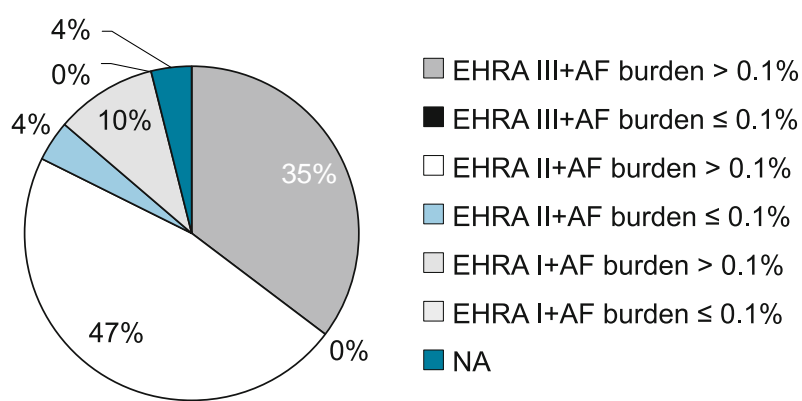

Fig. 9. Distribution of patients with $\geq 2 \mathrm{CA} A F$ according to the AF symptoms and AF burden after the first CA AF (EHRA - evaluation of symptoms related to $\mathrm{AF}, \mathrm{NA}$ - not available).

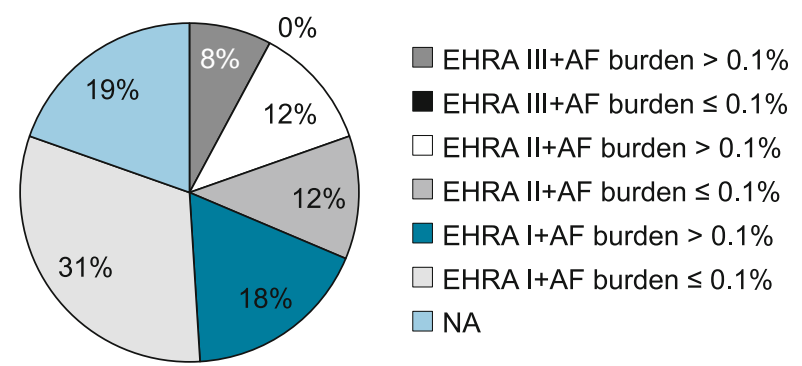

Fig. 10. Distribution of patients with $\geq 2$ CA AF according to the AF symptoms and $\mathrm{AF}$ burden after the last $\mathrm{CA} A \mathrm{AF}$.

In the group of patients undergoing a single procedure patients in groups EHRA III and with AF burden $>0.1 \%$ and EHRA III and AF burden $\leq 0.1 \%$ were the most represented ones before the procedure. After the procedure the most represented were the patients in EHRAI and AF burden $\leq 0.1 \%$, thereafter in group EHRA II with AF burden $>0.1 \%$ and in group EHRA I with AF burden $>0.1 \%$, respectively. Patients undergoing a single procedure were compared to patients in the work by Tondo (18). Patients in the presented study were selected based on the same inclusion criteria (implantation with a long-term ECG Holter monitor and undergoing one CAAF) and were divided into two groups. One group represented patients with recurrence of $\mathrm{AF}$ and the second $\mathrm{AF}$ patients without recurrence. For comparison purposes of our and Tondo's work, we compared patients with one recurrence in Tondo's study with patients with value of AF burden $>0.1 \%$ in our study. Next we compared patients without any recurrence in Tondo's study to patients with AF burden $\leq 0.1 \%$ in our study. The overall rate of the decline in symptoms after the procedure in the study was significant (from $82 \%$ before to $44 \%$ after the procedure) with reaching the same result in our study (from $90 \%$ to $43 \%$ ). In a more detailed analysis, we also found similar results. There were $54 \%$ of symptomatic patients with a single recurrence of symptoms in Tondo's study (compared to $34 \%$ in our group), $46 \%$ of asymptomatic patients (compared to $17 \%$ in our group), in Tondo's study in the group of patients without recurrence $29 \%$ of patients were symptomatic (compared to $9 \%$ in our group).

Evaluation of the second group: In the group of patients undergoing two or more performances we documented the highest 
$461-464$

proportion of patients with EHRA III and with AF burden $>0.1 \%$, followed by EHRA III and AF burden $\leq 0.1 \%$ before the first CA AF. In the mentioned group of patients we observed a decrease in the number of patients with EHRA III and AF burden $>0.1 \%$ and increase in the number of patients with EHRA II and AF burden $>$ $0.1 \%$ after the first CA AF. After the last realized procedure (CA AF) ( 2 and more realized procedures) the number of patients with EHRA I and AF burden $\leq 0.1 \%$ and at the same time EHRA I and AF burden $>0.1 \%$ increased.

On the basis of these results we can conclude that in the group of patients undergoing a single procedure the number of highly symptomatic patients (EHRA III) and the duration of AF burden were reduced significantly.

In a group of patients undergoing repeated procedures the reduction in the number of patients presenting themselves with significant symptoms (EHRA III) was significant when compared to data before the first procedure, but a significant reduction in the duration of $\mathrm{AF}$ ( $\mathrm{AF}$ burden $>0.1 \%$ ) is not present at the same time. If a given group of patients underwent a repeated procedure, we observed a progression in the withdrawal of symptoms (increasing the proportion of patients in group EHRA I) and reduction of the duration of $\mathrm{AF}$ ( $\mathrm{AF}$ burden $\leq 0.1 \%$ )

When comparing the final effect of the procedure (one CAAF vs. repeated CAAF) in our group of patients, we observed a better effect of treatment for patients undergoing 1 procedure in terms of symptom relief and reduction of AF burden. This phenomenon in our work can be explained by the fact that in our patient population, patients undergoing repeated procedures are patients with longer duration of AF (higher percentage of AF burden) and patients with a higher incidence of persistent AF history.

The advantage of long-term ECG monitoring in our study was the ability to detect and monitor patients who are at risk, particularly patients who are asymptomatic (EHRA I) and have an AF burden $>0.1 \%$. This advantage was with exception for the limitation of poor patient compliance in evaluating an exact follow-up documented also by Vasamreddy CR (19) (Figs 6, 7, 8, 9 and 10).

We conclude that CAAF is an effective non-pharmacological method of reducing AF burden and patient's symptoms related to $\mathrm{AF}$ at the same time.

\section{Learning points}

CAAF is an effective non-pharmacological treatment, which is used primarily for the treatment of $\mathrm{AF}$ in symptomatic patients with paroxysmal AF, with minimal structural heart disease in cases of resistance to antiarrhythmic therapy and patient preference.

The increase in asymptomatic episodes of AF after CA AF is clinically relevant.

The advantage of long-term ECG monitoring is the ability to detect and monitor patients who are at risk, particularly patients who are asymptomatic (EHRA I) and have an AF burden $>0.1 \%$ and is of considerable importance both for the clinical evaluation of ablation efficacy and for individualized clinical management of patients, especially with respect to appropriate antithrombotic therapy.

\section{References}

1. Benjamin EJ, Levy D, Vaziri SM, D'Agostino RB, Belanger AJ, Wolf PA. Independent risk factors for atrial fibrillation a population-based cohort: the Framingham Heart Study. JAMA 1994; 271 (11): 840-844.

2. Benjamin EJ, Wolf PA, D'Agostino RB, Silbershatz H, Kannelet WB, Levy D. Impact of Atrial Fibrillation on the Risk of Death: The Framingham Heart Study. Circulation 1998; 98 (10): 946-952.

3. Heeringa J, van der Kuip DA, Hofman A, Kors JA, van Herpen G, Stricker BH, Stijnen T, Lip GY, Witteman JC. Prevalence, incidence and lifetime risk of atrial fibrillation: the Rotterdam study. Eur Heart J 2006; 27 (8): 949-953.

4. CammAJ,LipGY,DeCaterinaRetal.2012 focused update of the ESC guidelines for the management of atrial fibrillation. Eur Heart J 2012; 33 (21): 2719-2747.

5. Camm AJ, Kirchhof P, Lip GY et al. Odporúčania pre manažment fibrilácie predsiení. Cardiology Lett 2011; 20 (1): 20-66.

6. Savelieva I, Camm AJ. Clinical relevance of silent atrial fibrillation: prevalence, prognosis, quality of life, and management. J Interv Card Electrophysiol 2000; 4: 369-382.

7. Israel CW, Grönefeld G, Ehrlich JR, Li YG, Hohnloser SH. Long-term risk of recurrent atrial fibrillation as documented by an implantable monitoring device: implications for optimal patient care. J Am Coll Cardiol 2004; 43 (1): 47-52.

8. Page RL, Tilsch TW, Connolly SJ, Schnell DJ, Marcello SR, Wilkinson WE, Prichett EL. Azimilide Supraventricular Arrhythmia Program (ASAP) Investigators. Asymptomatic or "silent" atrial fibrillation: frequency untreated patients and patients receiving azimilide. Circulation 2003; 107 (8): 1141-1145.

9. Page RL, Wilkinson WE, Clair WK, McCarthy EA, Pritchett EL. Asymptomatic arrhythmias patients with symptomatic paroxysmal atrial fibrillation and paroxysmal supraventricular tachycardia. Circulation 1994; 89: 224-227.

10. Hindricks G, Piorowski C, Tanner H, Kobza R, Gerds-Li JH, Carbucicchio C, Kottkamp H. Perception of Atrial Fibrillation Before and After Radiofrequency Catheter Ablation: Relevance of Asymptomatic Arrhythmia Recurrence. Circulation 2005; 112 (3): 307-313.

11. Pürerfellner $\mathbf{H}$, Aichinger $\mathbf{J}$, Martinek $M$ et al. Quantification of Atrial Tachyarrhythmia Burden with an Implantable Pacemaker Before and After Pulmonary Vein Isolation. Pacing Clin Electrophysiol 2004; 27 (9): 1277-1283.

12. Gerstenfeld EP, Guerra P, Sparks PB, Hattori K, Lesh MD. Clinical outcome after radiofrequency catheter ablation of focal atrial fibrillation triggers. J Cardiovasc Electrophysiol 2001; 12 (8): 900-908.

13. Berkowitsch A, Neumann T, Kurzidim $K$ et al. Comparison of generic health survey SF-36 and arrhythmia related symptom severity check list relation to post-therapy AF recurrence. Europace 2003; 5 (4): 351-355.

14. Berkowitsch A, Greiss H, Vukaljovic D et al. Usefulness of atrial fibrillation burden as a predictor for success of pulmonary vein isolation. Pacing Clin Electrophysiol 2005; 28 (12): 1292-1301.

15. Gill JS. How to perform pulmonary vein isolation. Europace 2004; 6 (2): 83-91.

16. Michaud GF, John R. Percutaneous Pulmonary Vein Isolation for Atrial Fibrillation Ablation. Circulation 2011; 123 (20): e596-e601

17. Oral H, Scharf C, Chugh A et al. Catheter ablation for paroxysmal atrial fibrillation: segmental pulmonary vein ostial ablation versus left atrial ablation. Circulation 2003; 108 (19): 2355-2360.

18. Tondo C, Tritto M, Landolina $M$ et al. Rhythm-symptom Correlation Patients on Continuous Monitoring After Catheter Ablation of Atrial Fibrillation. J Cardiovasc Electrophysiol 2014; 25 (2): 154-160.

19. Vasamreddy CR, Dalal D, Dong J et al. Symptomatic and Asymptomatic Atrial Fibrillation Patients Undergoing Radiofrequency Catheter Ablation. J Cardiovasc Electrophysiol 2006; 17 (2): 134-139.

Received August 19, 2014. Accepted November 17, 2014. 